\title{
Effectiveness of Kangaroo Mother Care in Reducing Morbidity and Mortality among Preterm Neonates on Nasal Continuous Positive Airway Pressure: A Randomized Controlled Trial
}

\author{
Catherine P. Ricero-Luistro, MD, ${ }^{1}$ Maria Esterlita T. Villanueva-Uy, MD, MSPH, ${ }^{2}$ \\ Aurora Gloria I. Libadia, $\mathrm{MD}^{2}$ and Socorro De Leon-Mendoza, $\mathrm{MD}^{3}$ \\ ${ }^{1}$ Batangas Medical Center \\ ${ }^{2}$ Philippine General Hospital, University of the Philippines Manila \\ ${ }^{3}$ Kangaroo Mother Care Foundation Philippines, Inc.
}

\begin{abstract}
Background. Prematurity is a major cause of neonatal death. Continuous positive airway pressure (CPAP) is the recognized initial intervention among preterm neonates in respiratory distress. Kangaroo mother care (KMC) may
\end{abstract} help improve neonatal outcomes.

Objective. To determine the effectiveness of $\mathrm{KMC}$ in reducing morbidity and mortality among preterm neonates on CPAP via RAM nasal cannula (nCPAP).

Methods. A prospective, non-blinded, randomized controlled trial was conducted on eligible preterm neonates requiring $\mathrm{nCPAP}$ due to respiratory distress. They were randomly allocated to either KMC $(n=35)$ or conventional care groups $(n=35)$. Outcome measures included duration of nCPAP and oxygen support, physiologic parameters, morbidity, mortality, and length of hospital stay.

Results. The durations of nCPAP and oxygen support were both significantly shorter in the KMC group. Morbidities (air leak syndrome, necrotizing enterocolitis, and late-onset sepsis) were also significantly lower in the KMC group. Although the mortality rate and the hospital stay were lower in the KMC group, these were not statistically significant.

Conclusion. KMC effectively decreased the duration of nCPAP and oxygen support, as well as the incidence of neonatal morbidities. There were trends towards reduced mortality and length of hospital stay in the neonates who received $\mathrm{KMC}$.

Key Words: Kangaroo Mother Care, Preterm Neonates, nasal CPAP, Oxygen Support

Paper presented (Oral) in the $2^{\text {nd }}$ World Congress on Pediatrics and Clinical Pediatrics June 2019, at Edinburgh, Scotland.

Poster presented in the $11^{\text {th }}$ Workshop and Congress on Kangaroo Mother Care on November 2016, at Trieste, Italy.

Poster presented in the Summer Conference on Neonatology on June 2015, at Provence, Avignon, France.

Paper presented (Oral) in the Annual Convention of the Philippine Society of Newborn Medicine on February 2015, Manila, Philippines.

Corresponding author: Catherine P. Ricero-Luistro, MD Batangas Medical Center

Bihi Road, Kumintang Ibaba, Batangas, Philippines

Email: cprluistro@gmail.com

\section{INTRODUCTION}

Respiratory distress syndrome (RDS) is a breathing disorder of premature babies caused by an inherent surfactant deficiency. ${ }^{1}$ Surfactant, which is an essential substance that maintains alveolar expansion, is produced with increasing gestation. RDS affects about $1-1.7 \%$ of all newborns but can approach 100\% among those born before 26 weeks of gestation. ${ }^{2,3}$ While there may be other causes of respiratory distress at birth among preterm neonates, RDS is the most common among them and is the leading cause of death among those prematurely born. Prompt attention by the pediatric resuscitation team is required for these high-risk, premature infants as a surfactant may 
be instilled down the infant's airways, either as soon as the premature baby is born or when RDS is diagnosed. The development of surfactant replacement therapy is by far, one of the greatest breakthroughs in the management of RDS. It resulted in a dramatic decline in mortality from nearly $100 \%$ to less than $10 \%{ }^{1}$

Advancements in neonatal care such as the introduction of more sophisticated mechanical ventilators and medical care have increased preterm survival in the 1960s and 1970s. Unfortunately, these infants develop complications of oxygen toxicity and barotrauma leading to a higher rate of bronchopulmonary dysplasia and retinopathy of prematurity. To circumvent these complications, management is now geared towards non-invasive respiratory support. The nasal continuous positive airway pressure (CPAP) support is recognized as the first line respiratory intervention among preterm neonates in respiratory distress, which can be applied even at the delivery room. ${ }^{4}$ Since the basic pathophysiology of RDS is generalized atelectasis, CPAP solves this problem by functional residual capacity stabilization, alveolar recruitment, and decreasing work of breathing. ${ }^{5}$

The Philippines ranks $8^{\text {th }}$ out of 184 countries in the number of babies born prematurely and ranks 17 th for the total number of deaths due to complications from preterm birth. ${ }^{5}$ In the Philippines, $48 \%$ of children who die under the age of 5 years are newborn infants with the leading cause of newborn deaths being prematurity (39\%). ${ }^{6}$ In 2011, 11,290 deaths were attributed to preterm complications, which amount to an equivalent of 31 newborn deaths every day. Despite advances in technology and availability of steroids and surfactants for the prevention and treatment of RDS respectively, deaths related to short gestation and low birth weight continue to increase in the Philippines and many countries around the world.

Preterm infants should be able to survive with access to simple and affordable care. ${ }^{5}$ Kangaroo Mother Care (KMC) is a simple, non-invasive, low technology but high-impact intervention to reduce preterm mortality. The introduction of KMC in the NICU of the hospital in Manila enabled more preterm neonates to survive. ${ }^{7}$ Although the prematurity rate showed increasing trends in the years 2004-2012 (101 to 157 preterm births per 1,000 live births) in the hospital, the adoption of $\mathrm{KMC}$ in 2012 has resulted in a decrease in preterm deaths in the unit.

The KMC position is the technique of placing the infant prone on the chest of the mother or father/another adult provider, skin-to-skin, in an upright position. Depending on the preterm neonate's clinical stability, KMC may be restricted to a few hours per day or maybe extended and provided, for as long as possible.

$\mathrm{KMC}$ empowers parents by allowing them to play a significant role in the care and survival of their baby within the NICU setting. KMC is an effective way to meet the baby's needs of warmth, breastfeeding, stimulation, and safety. ${ }^{8}$ Most importantly, there is strong evidence that the $\mathrm{KMC}$ intervention decreases the risk of dying among premature infants. ${ }^{9}$ For this reason, the technique is now practiced in many developed as well as developing countries. In both settings, KMC is offered at least two hours per session, to supplement incubator care of sick and less stable patients, and for as long as 24 hours for stable, growing preterm, small babies. While the mothers remain the best $\mathrm{KMC}$ providers for their babies, any other person can share the role of KMC provider. ${ }^{10}$

In a small study of Nimbalkar in 2012, preterm infants on nasal CPAP (Fischer and Paykel ${ }^{\circledR}$ with short binasal prongs) were either started on KMC on the first hour of life $(n=13)$ or not $(n=12)$; to determine its effect on the duration of CPAP support. Those on KMC were weaned from CPAP at a mean of 34.08 hours while those not on KMC were weaned slighter longer at 38.67 hours. This small study showed that KMC during CPAP was feasible and safe. ${ }^{11}$

In the study by Azevedo, KMC was used to promote mother-infant bonding for preterm infants on mechanical ventilation. ${ }^{12}$ Forty-three preterm infants with a mean gestational age at birth of $29.1 \pm 1.6$ weeks and a mean birth weight of $1.1334 \pm 2318 \mathrm{~g}$ were included. The preterm infants were longitudinally assessed for $90 \mathrm{mins}$ ( $15 \mathrm{~min}$ before, $60 \mathrm{~min}$ in $\mathrm{KMC}$, and $15 \mathrm{~min}$ after) during the study periods. These periods were compared, and the dependent variables: heart rate, oxygen saturation $\left(\mathrm{SpO}_{2}\right)$, axillary temperature and mean arterial blood pressure; were all significantly different. However, the differences were deemed not clinically pertinent. Still, the study demonstrated that KMC was a safe method for preterm infants on mechanical ventilation. ${ }^{12}$

In another study by Punasanvala in 2018, it is possible to give $\mathrm{KMC}$ in preterm infants from the first day of birth who are on respiratory support (CPAP). Preterm infants receiving $\mathrm{KMC}$ had fewer episodes of apnea, hypothermia, sepsis, and reduced mortality, though not significant. It was also beneficial in early weaning from assisted ventilation (CPAP). ${ }^{13}$

\section{GENERAL OBJECTIVE}

To determine the effectiveness of KMC in reducing morbidity and mortality rate among preterm neonates with gestational age $\leq 36$ weeks and weight between 1000 grams to 2000 grams, on nasal continuous positive airway pressure via RAM cannula.

\section{ABBREVIATION LIST}

- RDS - Respiratory Distress Syndrome

- nCPAP - Nasal Continuous Positive Airway Pressure using RAM cannula

- $\quad$ KMC - Kangaroo Mother Care

- LBW - Low Birth Weight

- $\quad \mathrm{NICU}$ - Neonatal Intensive Care Unit 


\section{DEFINITION OF TERMS}

- Low birth weight neonates - infants with birthweight $<2000$ grams.

- Kangaroo Mother Care position - the placement of the preterm infant, in an upright and prone position, skinto-skin on the mother's chest, in between her breasts.

- Respiratory Distress Syndrome - state of surfactant deficiency in preterm infants manifested as respiratory distress.

\section{METHODS}

\section{Study Design}

This study is a prospective, non-blinded, randomized controlled trial conducted at the Neonatal Intensive Care Unit (NICU) of the hospital.

\section{Study Population}

Eligible participants included all preterm neonates admitted at the NICU who are < 36 weeks gestation and weighing 1000-2000 grams, with Apgar scores > 7 at the $1^{\text {st }}$ and $5^{\text {th }}$ minute of life and requiring nasal CPAP support due to respiratory distress.

Excluded were neonates with major congenital abnormalities especially facial and gastrointestinal anomalies. Neonates with gestational age below 28 weeks and birth weight less than 1000 grams were also not included in the study.

\section{Random allocation}

After informed consent was obtained from the mother or father, the infant was randomly assigned to either KMC or conventional care. Assignments were placed inside brown opaque envelopes which were prepared before the start of the study.

\section{Intervention}

All parents of participants enrolled in the KMC group underwent orientation and training on how to properly provide KMC to their preterm babies while on nasal CPAP. They were also taught how to recognize danger signs such as apnea, cyanosis, and decreased movement. Before the $\mathrm{KMC}$ session, the parent's chest was wiped dry with a clean cloth. No skin disinfectant was used. The parent was then asked to perform handwashing and then don the KMC tube provided by the study. A clean gown was worn with the opening in front and over the $\mathrm{KMC}$ tube. The parent was then asked to sit on a chair that was positioned beside the infant's incubator (Figure 1).

The neonate, donned with a cap and diaper, was placed gently in an upright and prone position against the parent's chest. The tube blouse covered the body of the infant. The neonate's head was turned towards the CPAP machine side and the RAM cannula was adequately secured. The neonate's

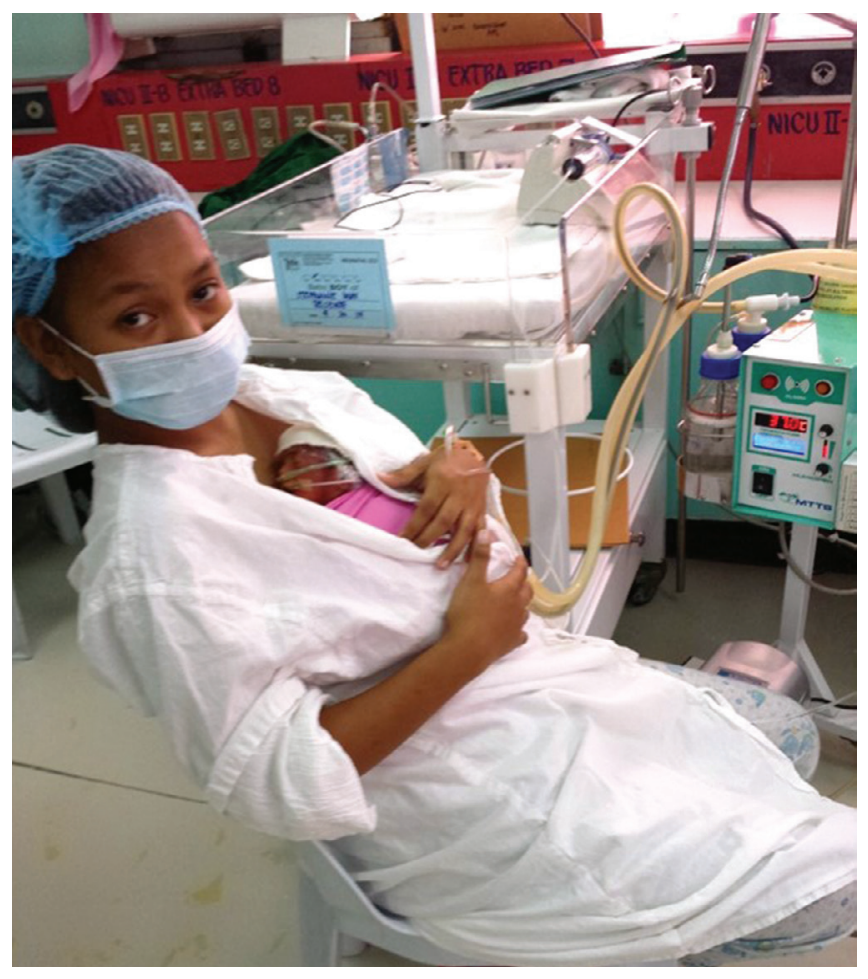

Figure 1. Mother providing $\mathrm{KMC}$ on her infant on RAM nasal cannula CPAP.

legs were in a "frog-like" position with his abdomen at the level of the parent's epigastrium. KMC was provided to all participants in the intervention group starting at 24 hours of life, intermittently for at least 4 hours daily until they were off nCPAP and oxygen support. Physiologic parameters such as heart rate, respiratory rate, temperature, oxygen saturation, and blood pressure were taken 15 minutes before, during, and 15 minutes after the $\mathrm{KMC}$ session. Clinical stability of the preterm in this context was defined as a heart rate of 120-160 beats per minute, respiratory rate of 40-60 cycles per minute, the temperature of 36.5-37.5 degrees Celsius (by digital thermometer), oxygen saturations $\geq 88 \%$, with or without inotropic support, measured for at least 15 minutes before the intervention.

When babies were not in KMC position, they were placed back inside their incubators during the study period.

\section{Conventional Care}

The participants in the control group were managed inside their incubators. Their vital signs, oxygen saturation by pulse oximetry, and thermal values by digital thermometer were recorded. Vital signs were obtained every hour between 6:00 pm to 10:00 pm, which was the time during which the NICU was deemed quietest and procedures most unobtrusive.

Clinical management remained the same between the two groups except for the provision of KMC to the intervention group. KMC provision was offered to both groups after the study period. 


\section{Statistical Analysis and Sample Size Estimation}

A total sample of 70 preterm neonates on nCPAP was calculated to achieve a power of $80 \%$ and an alpha error of 0.05. The mean difference of 3 days duration on mechanical ventilator support with a standard deviation of 4.3 was based on the study of Azevedo et al. ${ }^{12}$ Data were encoded using MS Excel program. SPSS version 16 statistical software was utilized for machine editing, data processing, and statistical analysis. The maternal and neonatal baseline characteristics were summarized using percentages, means, and standard deviations. Comparison of two means and three means were analyzed using T-test and ANOVA, respectively. A p-value of < 0.05 was considered significant.

\section{Ethical Considerations}

The protocol was approved by the Research Ethics Board.

\section{RESULTS}

A total of seventy preterm neonates admitted at the NICU of the hospital were included in the study. Thirtyfive (35) neonates each were randomly assigned to the $\mathrm{KMC}$ and the control groups.

Table 1 summarizes and compares the maternal and neonatal baseline characteristics between the $\mathrm{KMC}$ and control groups. Baseline maternal characteristics were not significantly different between the two groups except that there were significantly more obstetric complications among the KMC group compared with the control (45.7\% vs $22.9 \%$, $\mathrm{p}: 0.044)$. Neonatal characteristics were the same between the two groups except for significantly longer infants in the KMC group, although this was not clinically pertinent (40.34 vs. $40.31 \mathrm{~cm} ; \mathrm{p}=0.03)$. Also, the 5 -minute mean

Table 1. Maternal and neonatal baseline characteristics

\begin{tabular}{|c|c|c|c|}
\hline Characteristics & KMC (n=35) & Conventional Care $(n=35)$ & p-value \\
\hline Maternal Age in years (mean \pm SD) & $27.43 \pm 7.80$ & $28.20 \pm 7.46$ & 0.74 \\
\hline Maternal parity & $3(8.57)$ & $5(14.29)$ & 0.14 \\
\hline \multicolumn{4}{|l|}{ Presence of Maternal infection: $\mathrm{n}(\%)$} \\
\hline UTI & $13(37.14)$ & $12(34.29)$ & 0.63 \\
\hline Cough & $4(11.43)$ & $5(14.29)$ & 0.48 \\
\hline Preeclampsia & $6(17.14)$ & $7(20.00)$ & 0.48 \\
\hline Diabetes & $1(2.86)$ & $2(5.71)$ & 0.24 \\
\hline Obstetric Comorbidities n (\%) & $16(45.71)$ & $8(22.86)$ & 0.04 \\
\hline Medical comorbidities n (\%) & $9(25.71)$ & $6(17.14)$ & 0.08 \\
\hline Educational attainment & & & 0.39 \\
\hline Elementary & $2(5.71)$ & 0 & \\
\hline High school & $24(68.57)$ & $27(77.14)$ & \\
\hline College & $9(25.14)$ & $8(22.86)$ & \\
\hline Prenatal checkups (mean \pm SD) & $4.17 \pm 2.51$ & $4.37 \pm 2.58$ & 0.90 \\
\hline Mode of delivery: $\mathrm{n}(\%)$ & & & 0.60 \\
\hline Spontaneous vaginal delivery & $17(48.57)$ & $19(54.29)$ & \\
\hline Assisted vaginal delivery & 0 & 0 & \\
\hline Cesarean section & $18(51.43)$ & $16(45.71)$ & \\
\hline Male (in \%) & $18(51.43)$ & $18(51.43)$ & 0.85 \\
\hline Birthweight in grams (mean \pm SD) & $1559.43 \pm 330.20$ & $1551.29 \pm 287.82$ & 0.12 \\
\hline Birth length in $\mathrm{cm}$ (mean \pm SD) & $40.34 \pm 3.11$ & $40.31 \pm 2.34$ & 0.03 \\
\hline Gestational age in weeks (mean \pm SD) & $32.69 \pm 2.60$ & $32.34 \pm 2.22$ & 0.39 \\
\hline \multicolumn{4}{|l|}{ Apgar score (mean $\pm \mathrm{SD}$ ) } \\
\hline $1 \mathrm{~min}$ & $8.83 \pm 0.51$ & $8.37 \pm 1.19$ & 0.07 \\
\hline $5 \mathrm{~min}$ & $8.94 \pm 0.34$ & $8.62 \pm 0.94$ & 0.03 \\
\hline Respiratory diagnosis n (\%) & & & 0.68 \\
\hline Hyaline membrane disease & $19(54.29)$ & $19(54.29)$ & \\
\hline Transient tachypnea of the newborn & $7(20.00)$ & $8(22.86)$ & \\
\hline Neonatal pneumonia & $6(17.14)$ & $4(11.43)$ & \\
\hline HMD and pneumonia & $3(8.57)$ & $2(5.71)$ & \\
\hline TTN and pneumonia & 0 & $2(5.71)$ & \\
\hline
\end{tabular}

*T-test

HMD, Hyaline membrane disease; TTN, Transient tachypnea of the newborn 
Table 2. Comparison of outcomes between preterm neonates in the KMC group versus conventional care

\begin{tabular}{|c|c|c|c|}
\hline Outcomes & $\mathrm{KMC}(\mathrm{n}=35)$ & Conventional Care $(n=35)$ & p-value $(95 \% \mathrm{Cl})$ \\
\hline Mortality n (\%) & $2(5.71)$ & $3(8.57)$ & 0.36 \\
\hline No. of surfactant therapy & 0 & 0 & 0 \\
\hline No. of failed CPAP (invasive ventilation) & 0 & 0 & 0 \\
\hline Late-onset neonatal sepsis, n (\%) & $3(8.57)$ & $7(20.00)$ & 0.014 \\
\hline Nosocomial pneumonia, $\mathrm{n}(\%)$ & $2(5.71)$ & $3(8.57)$ & 0.477 \\
\hline Hypothermia, n (\%) & $2(5.71)$ & $10(28.57)$ & 0.36 \\
\hline Air leak syndromes, n (\%) & 0 & $1(2.86)$ & 0.043 \\
\hline Intraventricular hemorrhage, n (\%) & 0 & 0 & - \\
\hline Chronic lung disease, $\mathrm{n}(\%)$ & 0 & 0 & - \\
\hline Necrotizing enterocolitis, n (\%) & $2(5.71)$ & $5(14.28)$ & 0.016 \\
\hline Length of hospital stay (mean \pm SD) & $24.63 \pm 19.50$ & $28.14 \pm 19.48$ & 0.872 \\
\hline Discharge weight (mean \pm SD) & $1736.14 \pm 223.64$ & $1705.71 \pm 289.75$ & 0.596 \\
\hline Discharge length (mean \pm SD) & $41.01(2.85)$ & $40.94(2.57)$ & 0.257 \\
\hline
\end{tabular}

Apgar score was significantly higher in the KMC group $(8.94 \pm 0.34$ vs. $8.62 \pm 0.92 ; p=0.03)$ but again was not clinically relevant. The frequency of the different respiratory conditions for which nCPAP was required was comparable for both groups; with RDS being the most common etiology (Table 1).

With regards to neonatal outcomes, there was a trend for a lower neonatal mortality rate in the KMC group compared with the control $(5.71 \%$ vs. $8.57 \%)$ but this did not reach a statistical difference. Late-onset neonatal sepsis $(p=0.014)$, air leak syndromes $(p=0.043)$, and necrotizing enterocolitis ( $p=0.016$ ) were found to be significantly less in the KMC group. Hypothermia and nosocomial pneumonia were both less in the KMC group, but these were not statistically significant. Likewise, the participants in the KMC group appeared to have better growth parameters at discharge but were again not significantly different from those in the control group. Although not statistically significant, babies

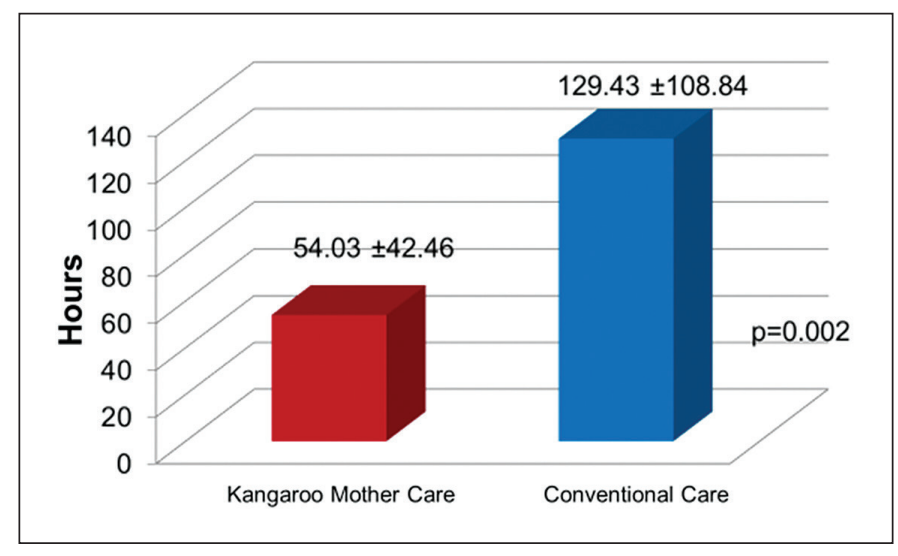

Figure 2. Comparison of duration of oxygen support between preterm neonates on $\mathrm{KMC}$ vs. conventional care. in the KMC group were discharged four days earlier than those in the control group (Table 2).

Analyses of the duration of respiratory support favor the $\mathrm{KMC}$ group. The mean duration of oxygen support (54.03 vs. 129.43 hours; $p=0.002$ ), and nasal CPAP (38.75 vs. 90.37 hours; $p=0.000$ ) were significantly shorter in the KMC compared with the control groups (Figures 2 and 3).

Physiologic parameters during the KMC session in the intervention group compared with the quiet sleep period in the control group showed significantly better thermoregulation and oxygen saturation in the intervention group (Table 3).

Likewise, a comparison of the physiologic parameters before, during, and after the KMC session in the intervention group also showed better thermoregulation during and after the KMC sessions. Systolic blood pressure was significantly lower (still within normal range) after the KMC session (Table 4).

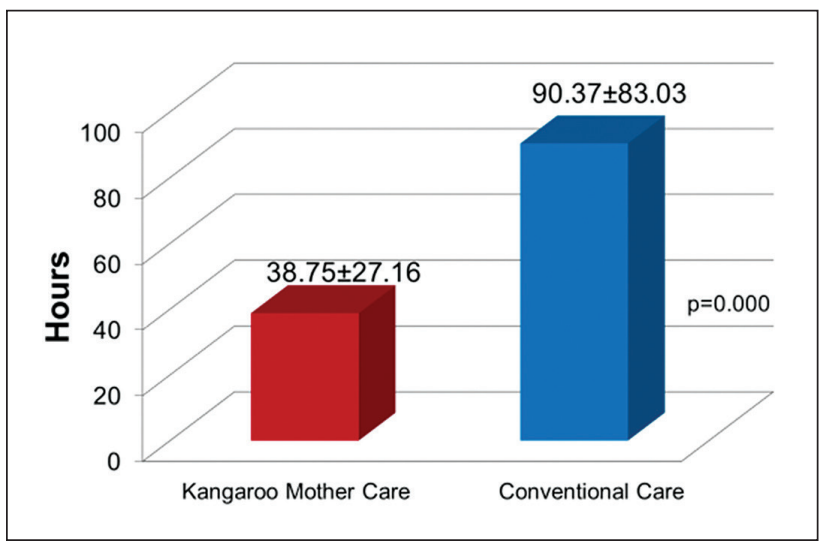

Figure 3. Comparison of duration of nasal CPAP via RAM cannula between preterm neonates on KMC vs. conventional care. 
Table 3. Comparison of physiologic parameters between infants on KMC versus conventional care

\begin{tabular}{|c|c|c|c|}
\hline \multirow{2}{*}{ Indicators } & $\mathrm{KMC}(\mathrm{n}=35)$ & Conventional Care $(n=35)$ & p-value \\
\hline & \multicolumn{3}{|c|}{ (Mean \pm SD) } \\
\hline Heart rate & $148.09 \pm 7.09$ & $148.37 \pm 10.12$ & 0.06 \\
\hline Respiratory rate & $55.11 \pm 8.23$ & $54.74 \pm 10.1$ & 0.16 \\
\hline Temperature & $36.56 \pm 0.27$ & $36.29 \pm 0.71$ & 0 \\
\hline Oxygen saturation & $95.94 \pm 2.11$ & $94.91 \pm 3.25$ & 0 \\
\hline Systolic blood pressure & $72.8 \pm 11.03$ & $65.75 \pm 7.85$ & 0.19 \\
\hline Diastolic blood pressure & $41.4 \pm 6.11$ & $37.75 \pm 4.11$ & 0.33 \\
\hline Capillary blood glucose & $125.24 \pm 40.71$ & $134 \pm 44.12$ & 0.20 \\
\hline
\end{tabular}

${ }^{*} T$-test

Table 4. Comparison of physiologic parameters before, during and after the KMC sessions in the KMC group

\begin{tabular}{lcccc}
\multicolumn{1}{c}{ Vital Signs } & $\mathbf{1 5}$ minutes before KMC & During KMC & 15 minutes after KMC & p-value \\
\hline Heart & $149.54 \pm 8.52$ & $148.09 \pm 7.09$ & $152.26 \pm 7.84$ & 0.103 \\
\hline Respiratory rate & $54.77 \pm 8.9$ & $55.11 \pm 8.23$ & $56.76 \pm 8.91$ & 0.593 \\
\hline Temperature & $36.23 \pm 0.59$ & $36.56 \pm 0.27$ & $36.66 \pm 0.36$ & 0.000 \\
\hline Oxygen saturation & $95.69 \pm 2.49$ & $95.94 \pm 2.11$ & $96.38 \pm 2.5$ & 0.471 \\
\hline Systolic BP & $69 \pm 15.8$ & $72.8 \pm 11.03$ & $63.07 \pm 9.83$ & 0.006 \\
\hline Diastolic BP & $39.5 \pm 10.09$ & $41.4 \pm 6.11$ & $37.03 \pm 6.23$ & 0.063 \\
\hline
\end{tabular}

${ }^{*}$ ANOVA

KMC, Kangaroo mother care; BP, Blood pressure

\section{DISCUSSION}

Recognizing the complications from invasive positive pressure ventilation, there has been a shift to non-invasive ventilation, specifically nasal CPAP. The use of the RAM nasal cannula hooked to CPAP or mechanical ventilator has been proven to be effective in delivering continuous or positive pressure to the respiratory tract with the infant being more comfortable. The RAM cannula has further allowed certain mobility to the baby's head and neck while receiving respiratory support.

$\mathrm{KMC}$ has long been proven to be effective in thermal control. ${ }^{14,15}$ Its use on all small babies as early as possible and for as long as possible has been recommended. ${ }^{16}$ The KMC method is superior to conventional care, particularly in reducing morbidities, especially in developing countries where resources are often limited. ${ }^{17}$ Currently, KMC is recommended for stable low birth weight infants.

$\mathrm{KMC}$ provision is slowly being shown to also be safe for preterm infants in respiratory distress and requiring respiratory support. The pilot study of Nimbalkar showed the feasibility and safety of KMC among infants on CPAP. In this study, there were fewer mortalities and morbidities among preterm infants on KMC compared with control. Air leaks, late-onset sepsis and NEC were significantly less in the KMC group. Hypothermia was again shown to be less frequent in the KMC group, although this was not statistically significant. ${ }^{11}$
In this study, there was a faster resolution of respiratory problems with the KMC group weaned from oxygen - 75 hours or roughly 3 days earlier than the control group. In the study of Nimbalkar, the KMC group was weaned from oxygen 4 hours earlier than those without KMC. ${ }^{11}$ Similarly, nCPAP was also weaned 51 hours or $>2$ days earlier in the KMC group compared with the control group. Providing $\mathrm{KMC}$ during non-invasive nCPAP treatment could have given additional comfort to an otherwise unpleasant experience for the newborns. This was evident with higher oxygen saturations in the KMC vs. the control group which could have led to faster weaning in oxygen and CPAP support. Similar data was shown in the study of Almeida in 2007 which concluded that KMC contributed significantly to the infants' physiological and respiratory improvements. ${ }^{18}$

As can be noted in the analysis of vital signs before, during, and after the KMC session, there was better thermoregulation during and after the $\mathrm{KMC}$ session. The systolic blood pressure was also lower after the KMC session. All these could indicate a more comfortable infant with less risk for cold stress. The effectiveness of KMC in this study on significantly reducing morbidities and duration of respiratory support were evident even when only intermittent KMC was provided at the $24^{\text {th }}$ hour of life and for only 4 hours/day. The potential for a much greater benefit may be seen if $\mathrm{KMC}$ is provided earlier and longer among these infants. In a study by Nyqvist, the kangaroo position also provides a neutral thermal environment that provides 
immature infants with optimal thermal regulation, which is the same or better than provided by an incubator. ${ }^{19}$

Conde-Agudelo ${ }^{14}$ and Dandekar ${ }^{17}$ showed that KMC led to better weight and subsequently shorter hospital stay. Although these parameters, on analyses, did not reach statistical significance, a shorter hospital stay of 4 days is equivalent to significant money saved, less risk for nosocomial infections, and earlier time to be with family.

\section{CONCLUSION}

KMC provided to preterm neonates on nasal CPAP significantly shortened the duration of oxygen support and RCPAP requirement. Furthermore, KMC contributed to a significant reduction in the incidence of morbidities such as air leak syndromes, necrotizing enterocolitis, and lateonset neonatal sepsis.

\section{Statement of Authorship}

All authors participated in the data collection and analysis and approved the final version submitted.

\section{Author Disclosure}

All authors declared no conflicts of interest.

\section{Funding Source}

The study was funded by the United States Agency for International Development (USAID) - Maternal and Child Health Integrated Program (MCHIP) 2013-2014.

\section{REFERENCES}

1. Rubarth LB, Quinn J. Respiratory development and respiratory distress syndrome. Neonatal Netw. 2015; 34(4):231-8. doi: 10.1891/ 0730-0832.34.4.231. PMID: 26802638.

2. Hermansen CL, Lorah KN. Respiratory distress in the newborn. Am Fam Physician. 2007 Oct 1; 76(7):987-94. PMID: 17956068.

3. Wambach A, Hamvas A. Respiratory distress syndrome in the neonate. In Martin RJ, Fanaroff AA, Walsh MC, eds. Fanaroff and Martin's Neonatal-Perinatal Medicine.10th ed. Philadelphia, PA: Elsevier Saunders; 2015. chap. 72.

4. Sweet DG, Carnielli V, Greisen G, Hallman M, Ozek E, Te Pas A, et al. European Consensus Guidelines on the Management of Respiratory Distress Syndrome - 2019 Update. Neonatology. 2019; 115(4):432-50. doi: 10.1159/000499361. PMID: 30974433.

5. March of Dimes, PMNCH, Save the Children, WHO. Born Too Soon: The Global Action Report on Preterm Birth. Eds. Howson CP, Kinney MV, Lawn JE. World Health Organization. Geneva, 2012 [Internet]. [cited 2014]. Available from: https://www.who.int/pmnch/ media/news/2012/201204_borntoosoon-report.pdf
6. WHO and UNICEF Countdown to 2015: Maternal, Newborn and Child Survival. Building a Future for Women and Children. The 2012 Report [Internet]. [cited 2014]. Available from: https://www. countdown2015mnch.org/documents/2012Report/2012-Complete. pdf

7. Section of Newborn Medicine Department of Pediatrics, University of the Philippines, Philippine General Hospital. Annual Perinatal Statistics. 2004-2012. Unpublished data

8. Tallandini MA, Scalembra C. Kangaroo mother care and motherpremature infant dyadic interaction. Infant Ment Health J. 2006 May; 27(3):251-75. doi: 10.1002/imhj.20091. PMID: 28640473.

9. Conde-Agudelo A, Belizán JM, Diaz-Rossello J. Kangaroo mother care to reduce morbidity and mortality in low birthweight infants. Cochrane Database Syst Rev. 2011 Mar 16;(3):CD002771. doi: 10.1002/14651858.CD002771.pub2. Update in: Cochrane Database Syst Rev. 2014;4:CD002771. PMID: 21412879.

10. Anderson GC, Chiu SH, Dombrowski MA, Swinth JY, Albert JM, Wada N. Mother-newborn contact in a randomized trial of kangaroo (skin-to-skin) care. J Obstet Gynecol Neonatal Nurs. 2003 Sep-Oct; 32(5):604-11. doi: 10.1177/0884217503256616. PMID: 14565739.

11. Nimbalkar S, Tandon R, Chaudhary N. Poster 1809. Reduced duration of CPAP in preterm babies receiving kangaroo care within an hour of birth - randomized trial. Arch Dis Child. 2012; 97:A512.

12. Azevedo VM, Xavier CC, Gontijo Fde O. Safety of kangaroo mother care in intubated neonates under 1500 g. J Trop Pediatr. 2012 Feb; 58(1):38-42. doi: 10.1093/tropej/fmr033. PMID: 21508081.

13. Punasanvala P, Parekh A, Gohil J, Pujari S. Kangaroo mother care in preterm baby receiving assisted respiratory support (CPAP): case-control trial. Int J Contemp Pediatr. 2018 May; 5(3):1008-12.

14. Conde-Agudelo A, Díaz-Rossello JL. Kangaroo mother care to reduce morbidity and mortality in low birthweight infants. Cochrane Database Syst Rev. 2016 Aug 23;2016(8):CD002771. doi: 10.1002/14651858.CD002771.pub4. PMID: 27552521.

15. Lawn JE, Mwansa-Kambafwile J, Horta BL, Barros FC, Cousens S. 'Kangaroo mother care' to prevent neonatal deaths due to preterm birth complications. Int J Epidemiol. 2010 Apr; 39 Suppl 1:i144-54. doi: 10.1093/ije/dyq031. PMID: 20348117.

16. Renjana RJ, Nayak S, Paul S. Comparison of radiant warmer care and kangaroo mother care shortly after birth on the neurobehavioral responses of the newborn. Journal of South Asian Federation of Obstetrics and Gynecology, January-April 2011; 3(1):53-5

17. Dandekar RH, Shafee M. Kangaroo Mother Care technology as a boon to tertiary care hospital in western Maharashtra. Int J Biomed Adv Res. Oct 2013; 4(10):731 doi:10.7439/ijbar.v4i10.502

18. Almeida CM, Almeida AFN, Forti EMP. Effects of Kangaroo mother care on the vital signs of low-weight preterm newborns. BrazJ Phys Ther. Jan-Feb 2007; 11(1):1-5. doi: 10.1590/S1413-35552007000100002

19. Nyqvist KH, Anderson GC, Bergman N, Cattaneo A, Charpak N, Davanzo R, et al. Towards universal Kangaroo Mother Care: recommendations and report from the First European conference and Seventh International Workshop on Kangaroo Mother Care. Acta Paediatr. 2010 Jun; 99(6):820-6. doi: 10.1111/j.16512227.2010.01787.x. PMID: 20219044 